\title{
Predictive Compensation for Wireless Networked System with Time Delay and Packet Dropout Based on T-S Model
}

\author{
Le Wang, Haipeng Pan, Jinfeng Gao, and Dongdong Chen \\ Department of Automation, Zhejiang Sci-Tech University, Hangzhou 310018, China \\ Correspondence should be addressed to Le Wang; lesley_wl@126.com
}

Received 10 July 2014; Accepted 11 August 2014; Published 26 August 2014

Academic Editor: Zhitao Liu

Copyright ( 2014 Le Wang et al. This is an open access article distributed under the Creative Commons Attribution License, which permits unrestricted use, distribution, and reproduction in any medium, provided the original work is properly cited.

\begin{abstract}
Based on the T-S model, a predictive compensation scheme including timer and counter for wireless networked system with long time delay and data packet dropout is proposed in this paper. By the separation principle, the state observation predictor and the state feedback controller are designed separately. For the case of fixed delay, the stability of the closed-loop networked control systems is discussed. Simulation by inverted pendulum system illustrates the effectiveness of the proposed method in wireless networked system based on T-S model.
\end{abstract}

\section{Introduction}

It has always been hot research on how to reduce the network-induced delays, packet dropouts, and other factors that affect the stability of the networked control systems and wireless sensor networks [1-3]. From the perspective of communication, [4] proposes an enhanced routing protocol on ad hoc on-demand distance vector with load balance and delay restriction, which can shorten the end-to-end delay and reduce the packet dropout. A new dynamic routing control strategy for wireless networks is proposed to monitor the inclination of multiple cranes in [5], which can reduce the measurement packet dropout. The issue of analysis and grey modeling for the time delay of star topology wireless networked control systems is researched in [6]. From the perspective of control theory, the state feedback controller, the state observer, and the observer-based output feedback controller are, respectively, derived for linear time-varying system in [7] to guarantee the asymptotic stability of the closed-loop system. In [8], the sufficient condition of the asymptotic stability for the singular pulse system is derived according to Lyapunov function theory, and the design method of the quantized feedback controller is presented. Networked control with reset quantized state has been, respectively, studied in $[9,10]$, where the former is observerbased, while the latter is based on Bernoulli processing.
Moreover, in [11], output feedback stabilization has been analyzed for Markov-based nonuniformly sampled-data networked control systems. And in [12], mixed time delays in stochastic Markovian jump neural networks have been explored in depth. Similarly, in [13] the problem of sampleddata synchronization for Markovian jump neural networks with time-varying delay and variable samplings is considered. In [14], a simplified model predictive control algorithm is designed for discrete-time Markov jump systems with mixed uncertainties. Although there are a lot of literatures for the networked linear systems, nonlinear systems, continuous systems, discrete systems, time-varying systems, time-invariant systems, or any combination of the several systems described above [15-17], which have made some achievements, time delay and packet dropout rate are rarely real-time computed while designing the controller and compensator. Therefore, referring to the model of [18] and design scheme in [19], this paper proposes a method with timers and counters which can not only calculate time delay and packet dropout rate in real time but also give the effective predictive compensation.

\section{Problem Formulation}

The framework of the system over a network medium researched in this paper is shown in Figure 1. The sensor, 


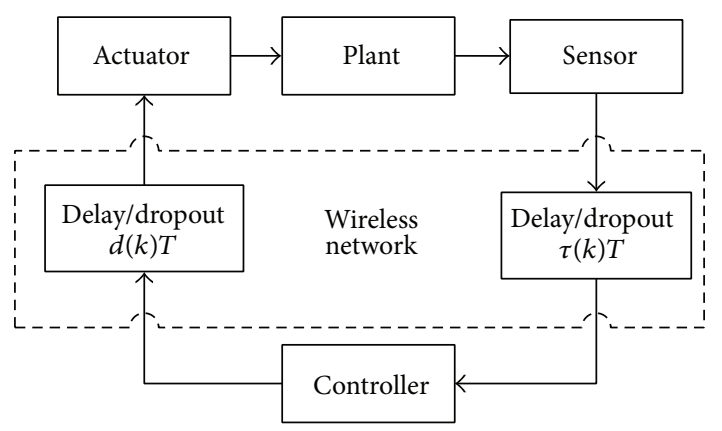

FIgURE 1: The structure of wireless networked control system.

the controller, and the actuator reside in physically different nodes communicating over shared communication networks. Due to the limited network bandwidth, unavoidable network congestion as well as network interruption, network transmission delay, and packet dropout during information exchange between different components appear.

For convenience, some general assumptions are given as follows.

(1) The node of sensor is time-driven, and the sampling period denoted by $T$ is fixed; the controller and the actuator are event-driven and execute the appropriate action, respectively, when data packets arrive [20].

(2) There is no time delay or packet dropout during a sampling period. And assume that the data are lost if the transmission time exceeds the maximum transmission delay, which is set as $n T$.

(3) The time delay and packet dropout between the sensor and the controller are marked as $\tau(k) T$, while between the sensor and the controller they are marked as $d(k) T$, including computation delay in each module.

(4) The output data from the sensor and the controller have a time stamp and are transmitted with a single packet without wrong order. form:

Consider a plant described in the following state-space

$$
\begin{gathered}
x(k+1)=A x(k)+B u(k) \\
y(k)=C x(k) \\
u(k)=K x(k),
\end{gathered}
$$

where $x(k) \in R^{n}, u(k) \in R^{m}$, and $y(k) \in R^{q}$ are the state vector, control input, and output vector, respectively. $A, B, C$, and $K$ are matrices with appropriate dimensions.

According to [18, 21], system (1) mentioned above can be described by the IF-THEN fuzzy rule as follows.

Rule i. Consider

$$
\begin{gathered}
R_{i}: \text { IF } \theta_{1}(k) \text { is } M_{i 1} \text { and } \cdots \text { and } \theta_{n}(k) \text { is } M_{i n} \\
\text { THEN } x(k+1)=A_{i} x(k)+B_{i} u(k), \\
y(k)=C_{i} x(k),
\end{gathered}
$$

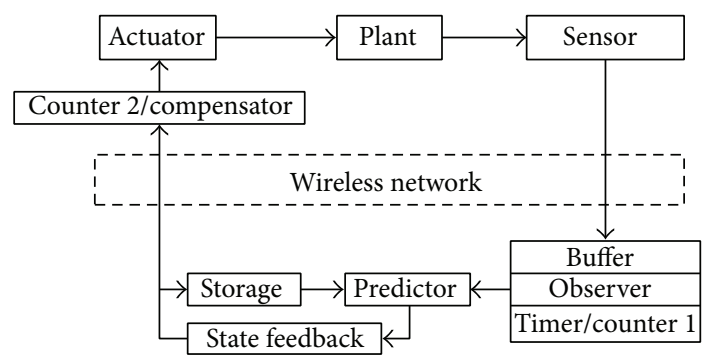

FIGURE 2: Wireless networked system digraph with predictor.

where $i=1,2, \ldots, r, r$ is the number of IF-THEN rules, $\theta_{1}(k), \theta_{2}(k), \ldots, \theta_{n}(k)$ are the premise variables, $M_{i 1}, M_{i 2}, \ldots, M_{i n}$ are fuzzy sets, and $A_{i}, B_{i}, C_{i}$ are matrices with appropriate dimensions.

By using the fuzzy inference method with a singleton fuzzifier, product inference, and center average defuzzifier, we can get the global fuzzy equation of the $i$ th rule:

$$
\begin{aligned}
x(k+1) & =\sum_{i=1}^{r} \lambda_{i}(\theta(k))\left[A_{i} x(k)+B_{i} u(k)\right] \\
y(k) & =\sum_{i=1}^{r} \lambda_{i}(\theta(k)) C_{i} x(k),
\end{aligned}
$$

where $\theta(k)=\left[\theta_{1}^{T}(k), \theta_{2}^{T}(k), \ldots, \theta_{n}^{T}(k)\right]^{T}, \quad \lambda_{i}(\theta(k))=$ $\prod_{j=1}^{n} M_{i j}\left(\theta_{j}(k)\right) / \sum_{i=1}^{r} \prod_{j=1}^{n} M_{i j}\left(\theta_{j}(k)\right), \lambda_{i}(\theta(k)) \geq 0$, $\sum_{i=1}^{r} \lambda_{i}(\theta(k))=1$, and $M_{i j}(\theta(k))$ is the membership function of fuzzy sets $M_{i j}$.

\section{Design for Wireless Networked Control System Based on T-S Model}

In real networked control system, limited by environmental or economic conditions, not all state variables are measurable. Based on hierarchical control structure, the design of networked control system with local state observer is presented in [22]. It introduces local state observer in the plant terminal and proceeds feedback control with state estimation at the end of the remote controller. As a consequence, this paper uses the state observer between the sensor and the controller. At the same time, in order to avoid data confusion and compute packet dropout rate over a period of time in real time, the data buffer and timing counter are added. It will adjust automatically when the packet dropout rate is bigger than the rate which is set to ensure the stability of the network. The networked control system designed in this paper is shown in Figure 2.

3.1. Buffer/Storage and Counter. The buffer and memory are used for temporary storage of data, which can filter data and avoid the wrong order. And the main purpose of the counters is to calculate the packet dropout.

Between the sensor and the controller, define two variables, $C_{S 1}$ and $C_{S 2}$. Consider $T$ as the timing unit. Within a sampling period, when time is up, $C_{S 2}$ will be increased by 
one. Meanwhile, if the data in the buffer are not changed at the moment, it will be $C_{S 1}=C_{S 1}+1$; otherwise, it will be $C_{S 1}=C_{S 1}$.

Similarly, between the controller and the actuator, define two variables, $C_{C 1}$ and $C_{C 2}$. Consider $T$ as the timing unit. Within a sampling period, when time is up, $C_{C 2}$ will be increased by one. Meanwhile, if the data in the buffer are not changed at the moment, it will be $C_{C 1}=C_{C 1}+1$; otherwise, $C_{C 1}=C_{C 1}$.

Networked control system can bear a certain degree of packet dropout. Hence, computing packet dropout rate currently will help ensure the stability of the system. The packet dropout rate $\gamma$ can be estimated by formula (5) in the improved networked control system mentioned above. For example, suppose that the data in the buffer have not been changed while the data in the memory have been changed within the time $T$, so $C_{S 1}=1, C_{S 2}=1, C_{C 1}=0$, and $C_{C 2}=1$. Then, within the time $2 T$, suppose that the data in the buffer and memory both have not been changed, so $C_{S 1}=2, C_{S 2}=2, C_{C 1}=1$, and $C_{C 2}=2$. Then, within the time $3 T$, suppose that the data in the buffer and memory both have been changed, so $C_{S 1}=2, C_{S 2}=3, C_{C 1}=1$, and $C_{C 2}=3$. Then, within the time $4 T$, suppose that the data in the buffer and memory both have been changed, so $C_{S 1}=2, C_{S 2}=4$, $C_{C 1}=1$, and $C_{C 2}=4$. Consequently, on the basis of (5), it follows that, at time $T, \gamma$ is 0.5 , at time $2 T, \gamma$ is 0.75 , at time $3 T, \gamma$ is 0.5 , and, at time $4 T, \gamma$ is 0.375 . Consider

$$
\gamma=\frac{1}{2}\left(\frac{C_{S 1}}{C_{S 2}}+\frac{C_{C 1}}{C_{C 2}}\right) .
$$

3.2. The State Observer. For arbitrary initial value of system (1) and the state observer, $\widehat{x}(n)$ will be equal to $x(n)$ after $n$ step at most. That is to say, estimated values and state values are equal. However, the continuous system has no such properties [23].

Assume that the current moment is $k$. And then make a comparison between the time stamp and local time so that $\tau(k) T$ is acquired. Next, round $\tau(k)$. If the buffer data are updated at the moment, $\tau(k)$ is less than or equal to $\tau(k-1)$. In general, use the output and input of the plant as an input of the state observer. The feedback from the controller is $(y(k-\tau(k)), u(k-\tau(k)))$. A state observer for system (4) is structured as follows [24]:

$$
\begin{aligned}
& \widehat{x}(k+1-\tau(k))=\sum_{i=1}^{r} \lambda_{i}(\theta(k)) \\
& \times\left[A_{i} \widehat{x}(k-\tau(k))+B_{i} u(k-\tau(k))\right. \\
&\left.\quad+L_{i}(y(k-\tau(k))-\widehat{y}(k-\tau(k)))\right] \\
& \widehat{y}(k-\tau(k))=\sum_{i=1}^{r} \lambda_{i}(\theta(k)) C_{i} \hat{x}(k-\tau(k)),
\end{aligned}
$$

where $\widehat{x}(k) \in R^{n}, \widehat{y}(k) \in R^{q}$ are the state and output vector of the state observer, respectively, and $L_{i} \in R^{n \times q}$ is the state observer gain, which can be obtained through dual principle.
3.3. The Predictor. As we all know, the packet dropout can also be converted to time delay. Values at current moment cannot be acquired because of the network-induced delay. Even so, $y(k-\tau(k))$ can be acquired. Then, combine $y(k-$ $\tau(k))$ and $\widehat{x}(k+1-\tau(k))$; the system state values from $k+2-$ $\tau(k)$ to $k$ can be further predicted as follows:

$$
\begin{aligned}
& \widehat{x}(k+2-\tau(k))=\sum_{i=1}^{r} \lambda_{i}(\theta(k))\left[A_{i} \widehat{x}(k+1-\tau(k))\right.\left.+B_{i} u(k+1-\tau(k))\right] \\
& \widehat{x}(k+3-\tau(k))=\sum_{i=1}^{r} \lambda_{i}(\theta(k))\left[A_{i} \widehat{x}(k+2-\tau(k))\right. \\
&\left.+B_{i} u(k+2-\tau(k))\right] \\
& \vdots \\
& \widehat{x}(k-1)=\sum_{i=1}^{r} \lambda_{i}(\theta(k))\left[A_{i} \widehat{x}(k-2)+B_{i} u(k-2)\right] \\
& \widehat{x}(k)=\sum_{i=1}^{r} \lambda_{i}(\theta(k))\left[A_{i} \widehat{x}(k-1)+B_{i} u(k-1)\right] .
\end{aligned}
$$

From the above formulas, the relationship between $\widehat{x}(k)$ and $\widehat{x}(k+1-\tau(k))$ can be built by recursion; that is,

$$
\begin{aligned}
\widehat{x}(k)= & \left(\sum_{i=1}^{r} \lambda_{i}(\theta(k)) A_{i}\right)^{\tau(k)-1} \widehat{x}(k+1-\tau(k)) \\
& +\sum_{j=1}^{\tau(k)-1}\left(\sum_{i=1}^{r} \lambda_{i}(\theta(k)) A_{i}\right)^{j-1} \\
& \times\left(\sum_{i=1}^{r} \lambda_{i}(\theta(k)) B_{i}\right) u(k-j) .
\end{aligned}
$$

3.4. The State Feedback Controller. There exist random delay and packet dropout between the controller and the actuator, marked as $d(k) T$. In order to keep the controller synchronized with the plant, it should calculate the controlled quantity $u_{k+l}$, where $l=0,1, \ldots, d$.

The state feedback controller is designed as formula (9), and $\widehat{x}(k+l)$ is the estimate value when the time delay is $\tau(k) T$. Consider

$$
u_{k+l}=\sum_{i=1}^{r} \lambda_{i}(\theta(k))\left(K_{i} \widehat{x}(k+l)\right) \text {, }
$$

where $l=0,1, \ldots, d$ and $K_{i} \in R^{m \times q}$ is a state feedback controller gain. 
By formulas (7) (9), the system state values from $k+1$ to $k+d$ can also be further predicted, which is as follows

$$
\begin{gathered}
\widehat{x}(k+l)=\left(\left(\sum_{i=1}^{r} \lambda_{i}(\theta(k)) A_{i}\right)+\left(\sum_{i=1}^{r} \lambda_{i}(\theta(k)) B_{i}\right)\right. \\
\left.\times \sum_{i=1}^{r} \lambda_{i}(\theta(k)) K_{i}\right)^{l} \widehat{x}(k) .
\end{gathered}
$$

Substituted into the formula (9), it follows that

$$
\begin{aligned}
& u_{k+l}=\sum_{i=1}^{r} \lambda_{i}(\theta(k)) \\
& \times\left\{K _ { i } \left[\left(\sum_{i=1}^{r} \lambda_{i}(\theta(k)) A_{i}\right)+\left(\sum_{i=1}^{r} \lambda_{i}(\theta(k)) B_{i}\right)\right.\right. \\
&\left.\left.\left.\times \sum_{i=1}^{r} \lambda_{i}(\theta(k)) K_{i}\right)^{l} \times \hat{x}(k)\right]\right\} .
\end{aligned}
$$

Construct $u_{k+l}, l=0,1, \ldots, d$, as a control sequence $U_{k}=\left\{u_{k}, u_{k+1}, \ldots, u_{k+d}\right\}$, which will be packaged into packets together with the time stamp and then sent to the compensator.

3.5. The Compensator. Comparing the time stamp with local time or by the counter, we can achieve $d(k) T$. After that, round $d(k)$. It should be noticed that the control sequence received by the compensator is generated at the moment $k-d(k)$, but now, between the sensor and the controller, the time delay value is $\tau(k-d(k)) T$. For this reason, at time $k$, the total delay in the closed-loop network is not $(d(k)+\tau(k)) T$, but $(d(k)+\tau(k-d(k))) T$. Thus, the input and output of the plant are $u(k-d(k)-\tau(k-d(k)))$ and $y(k-d(k)-\tau(k-d(k)))$. Then combined with formula (10), the state estimator for $(d(k)+\tau(k-d(k))) T$ can be deserved as follows:

$$
\begin{aligned}
\widehat{x}(k-d(k)+l)=( & \left(\sum_{i=1}^{r} \lambda_{i}(\theta(k)) A_{i}\right)+\left(\sum_{i=1}^{r} \lambda_{i}(\theta(k)) B_{i}\right) \\
& \left.\times \sum_{i=1}^{r} \lambda_{i}(\theta(k)) K_{i}\right)^{l} \widehat{x}(k-d(k)) .
\end{aligned}
$$

With (12), one obtains

$$
\begin{gathered}
\widehat{x}(k)=\left(\left(\sum_{i=1}^{r} \lambda_{i}(\theta(k)) A_{i}\right)+\left(\sum_{i=1}^{r} \lambda_{i}(\theta(k)) B_{i}\right)\right. \\
\left.\times \sum_{i=1}^{r} \lambda_{i}(\theta(k)) K_{i}\right)^{d(k)} \widehat{x}(k-d(k)) .
\end{gathered}
$$

Thus, the control law now is

$$
\begin{aligned}
& u_{k}= \sum_{i=1}^{r} \lambda_{i}(\theta(k)) K_{i} \widehat{x}(k) \\
&=\sum_{i=1}^{r} \lambda_{i}(\theta(k)) K_{i} \\
& \quad \times\left(\left(\sum_{i=1}^{r} \lambda_{i}(\theta(k)) A_{i}\right)+\left(\sum_{i=1}^{r} \lambda_{i}(\theta(k)) B_{i}\right)\right. \\
&\left.\quad \times \sum_{i=1}^{r} \lambda_{i}(\theta(k)) K_{i}\right)^{d(k)} \\
& \quad \times \widehat{x}(k-d(k)) .
\end{aligned}
$$

At time $k$, the control sequence in the compensator described above can be set as $U_{k-d(k)}=$ $\left\{u_{k-d(k)}, u_{k-d(k)+1}, \ldots, u_{k}\right\}$. The control input would be $u_{k}$ in case of time delay compensation.

\section{Stability Analysis}

Define the following error vector:

$$
e(k)=x(k)-\widehat{x}(k)
$$

and define the following augmented vector:

$$
\phi(k)=\left[\begin{array}{l}
x(k) \\
e(k)
\end{array}\right] .
$$

In addition, set $A^{*}=\sum_{i=1}^{r} \lambda_{i}(\theta(k)) A_{i}, B^{*}=\sum_{i=1}^{r} \lambda_{i}(\theta(k)) B_{i}$, and $K^{*}=\sum_{i=1}^{r} \lambda_{i}(\theta(k)) K_{i}$.

(1) When $\tau(k+1)=\tau(k)+1$, there is $\widehat{x}(k+2-\tau(k-1))=$ $\widehat{x}(k+1-\tau(k))$.

Then combined with formula (8), it follows that

$$
\begin{aligned}
\widehat{x}(k+1)= & A^{* \tau(k)-1} \widehat{x}(k+2-\tau(k+1)) \\
& +\sum_{j=1}^{\tau(k+1)-1} A^{* j-1} B^{*} u(k+1-j) \\
= & A^{* \tau(k)} \widehat{x}(k+1-\tau(k)) \\
& +\sum_{j=1}^{\tau(k)} A^{* j-1} B^{*} u(k+1-j) \\
= & A^{* \tau(k)} A^{* 1-\tau(k)} \\
& \times\left[\widehat{x}(k)-\sum_{j=1}^{\tau(k)-1} A^{* j-1} B^{*} u(k-j)\right] \\
& +\sum_{j=1}^{\tau(k)} A^{* j-1} B^{*} u(k+1-j) \\
= & A^{*} \widehat{x}(k)+B^{*} u(k) .
\end{aligned}
$$


By formula (4), formula (15), and formula (17), we can get

$$
\begin{aligned}
e(k+1)= & x(k+1)-\widehat{x}(k+1) \\
= & A^{*} x(k)+B^{*} u(k) \\
& -\left(A^{*} \widehat{x}(k)+B^{*} u(k)\right) \\
= & A^{*} e(k) .
\end{aligned}
$$

Therefore,

$$
\begin{array}{r}
e(k)=A^{*} e(k-1) \\
e(k-1)=A^{*} e(k-2)
\end{array}
$$

Then,

$$
e(k-1)=A^{*-1} e(k) .
$$

Thus, with (19) and (20), it follows that

$$
e(k-d(k))=A^{*-d(k)} e(k) \text {. }
$$

And then, with (4), (14), (15), and (21), we obtain $x(k+1)=A^{*} x(k)$

$$
\begin{aligned}
& +B^{*} K^{*}\left(A^{*}+B^{*} K^{*}\right)^{d(k)} \\
& \times[x(k-d(k))-e(k-d(k))] \\
= & A^{*} x(k)+B^{*} K^{*}\left(A^{*}+B^{*} K^{*}\right)^{d(k)} \\
& \times\left[\left(A^{*}+B^{*} K^{*}\right)^{-d(k)} x(k)-A^{*-d(k)} e(k)\right] \\
= & \left(A^{*}+B^{*} K^{*}\right) x(k)-B^{*} K^{*}\left(A^{*}+B^{*} K^{*}\right)^{d(k)} \\
& \times A^{*-d(k)} e(k) .
\end{aligned}
$$

Then, on the basis of formulas (16), (18), and (22), we get $\phi(k+1)$

$$
\begin{aligned}
& =\left[\begin{array}{l}
x(k+1) \\
e(k+1)
\end{array}\right] \\
& =\left[\begin{array}{cc}
\left\{A^{*}+B^{*} K^{*}\right\} & \left\{-B^{*} K^{*}\left(A^{*}+B^{*} K^{*}\right)^{d(k)} \times A^{*-d(k)}\right\} \\
0 & A^{*}
\end{array}\right] \\
& \times\left[\begin{array}{c}
x(k) \\
e(k)
\end{array}\right] .
\end{aligned}
$$

$$
\begin{aligned}
e(k+1)= & x(k+1)-\hat{x}(k+1) \\
= & A^{*} x(k)+B^{*} u(k) \\
& -\left(A^{*} \hat{x}(k)+B^{*} u(k)+L^{*} C^{*}(x(k)-\widehat{x}(k))\right) \\
= & \left(A^{*}-L^{*} C^{*}\right) e(k) .
\end{aligned}
$$

Therefore,

$$
e(k-d(k))=\left(A^{*}-L^{*} C^{*}\right)^{-d(k)} e(k) .
$$

By formula (4) and formula (26), we can achieve

$$
\begin{aligned}
x(k+1)= & A^{*} x(k)+B^{*} K^{*}\left(A^{*}+B^{*} K^{*}\right)^{d(k)} \\
& \times[x(k-d(k))-e(k-d(k))] \\
= & A^{*} x(k)+B^{*} K^{*}\left(A^{*}+B^{*} K^{*}\right)^{d(k)} \\
& \times\left[\left(A^{*}+B^{*} K^{*}\right)^{-d(k)} x(k)\right. \\
& \left.-\left(A^{*}-L^{*} C^{*}\right)^{-d(k)} e(k)\right] \\
& -B^{*} K^{*}\left(A^{*}+B^{*} K^{*}\right)^{d(k)} \\
& \times\left(A^{*}-L^{*} C^{*}\right)^{-d(k)} e(k) .
\end{aligned}
$$

Then with (16), (25), and (27), it follows that 


$$
\begin{aligned}
& \phi(k+1)=\left[\begin{array}{l}
x(k+1) \\
e(k+1)
\end{array}\right] \\
& =\left[\begin{array}{cc}
\left\{A^{*}+B^{*} K^{*}\right\} & \left\{-B^{*} K^{*}\left(A^{*}+B^{*} K^{*}\right)^{d(k)} \times\left(A^{*}-L^{*} C^{*}\right)^{-d(k)}\right\} \\
0 & A^{*}-L^{*} C^{*}
\end{array}\right] \\
& \times\left[\begin{array}{l}
x(k) \\
e(k)
\end{array}\right] .
\end{aligned}
$$

As we know, if all the eigenvalues of the matrix $\quad\left[\begin{array}{c}A^{*}+B^{*} K^{*}-B^{*} K^{*}\left(A^{*}+B^{*} K^{*}\right)^{d(k)} A^{*-d(k)} \\ 0\end{array}\right] \quad A^{*} \quad$ and $\left[\begin{array}{c}A^{*}+B^{*} K^{*}-B^{*} K^{*}\left(A^{*}+B^{*} K^{*}\right)^{d(k)}\left(A^{*}-L^{*} C^{*}\right)^{-d(k)} \\ A^{*}-L^{*} C^{*}\end{array}\right]$ are located in the open unit disc, the system will be asymptotically stable, even there are $\tau(k) T$ and $d(k) T$. The separation principle still holds in discrete systems. It indicates that the closed-loop system with state feedback still retains the original nature of the state feedback closed-loop system after the access to the observer. So the pole assignment can be separated into two parts: for state feedback and for the observer [23]. Characteristic polynomials of the two matrices introduced above are, respectively, $\operatorname{det}\left(A^{*}+B^{*} K^{*}\right) \operatorname{det} A^{*}$ and $\operatorname{det}\left(A^{*}+B^{*} K^{*}\right) \operatorname{det}\left(A^{*}-L^{*} C^{*}\right)$. Obviously, the controller gain and the state observer gain can be conducted based on the separation principle.

\section{Numerical Example}

Consider an inverted pendulum system [25]. Suppose sample time $T=0.1 \mathrm{~s}$, and select the following state variables:

$$
x(t)=[\phi(t), \dot{\phi}(t)]^{T}=\left[\begin{array}{ll}
x_{1}, & x_{2}
\end{array}\right]^{T},
$$

where $\phi(t)$ signifies an angle that is between the pendulum and the vertical line and $\dot{\phi}(t)$ signifies an angular velocity. Linearize the original inverted pendulum model at five working points, $0^{\circ}, \pm 15^{\circ}$, and $\pm 30^{\circ}$. Then use formula (3) in [26] to discretize it, so we can get matrix coefficients $A_{i}$ and $B_{i}$.

Then, set $C_{i}=C=\left[\begin{array}{ll}1 & 0\end{array}\right]$.

As a result, the T-S fuzzy model can be written as follows:

$$
\begin{aligned}
& R_{1}: \operatorname{IF} \quad \theta_{1}(k) \text { is about } 0, \\
& \text { THEN } x(k+1)=A_{1} x(k)+B_{1} u(k), \\
& y(k)=C_{1} x(k),
\end{aligned}
$$

where

$$
A_{1}=\left[\begin{array}{ll}
1.0000 & 1.1052 \\
4.8997 & 1.0000
\end{array}\right], \quad B_{1}=\left[\begin{array}{l}
-.85317 e-2 \\
-.81100 e-2
\end{array}\right]
$$

By using rank criteria of system controllability and observability, the system is completely controllable and completely observable. Consider

$$
\begin{aligned}
& R_{2}: \operatorname{IF} \quad \theta_{1}(k) \text { is about } \pm 15^{\circ}, \\
& \text { THEN } x(k+1)=A_{2} x(k)+B_{2} u(k), \\
& y(k)=C_{2} x(k)
\end{aligned}
$$

where

$$
A_{2}=\left[\begin{array}{ll}
1.0000 & 1.1052 \\
4.5278 & 1.0000
\end{array}\right], \quad B_{2}=\left[\begin{array}{l}
-.81951 e-2 \\
-.77900 e-2
\end{array}\right]
$$

and the system now is also completely controllable and completely observable. Consider

$$
\begin{aligned}
& R_{3}: \text { IF } \theta_{1}(k) \text { is about } \pm 30^{\circ}, \\
& \text { THEN } x(k+1)=A_{3} x(k)+B_{3} u(k), \\
& y(k)=C_{3} x(k),
\end{aligned}
$$

where

$$
A_{3}=\left[\begin{array}{ll}
1.0000 & 1.1052 \\
3.6522 & 1.0000
\end{array}\right], \quad B_{3}=\left[\begin{array}{l}
-.72378 e-2 \\
-.68800 e-2
\end{array}\right]
$$

and the system now is also completely controllable and completely observable.

The controller poles are assigned to $0.3 \pm 0.5 j$ so that $K_{1}, K_{2}$, and $K_{3}$ could be achieved under the three kinds of preconditions described above. Three state observer gains would be obtained in the same way. In this simulation, the output variables can reflect the first state variables since $C_{i}=$ $C=\left[\begin{array}{ll}1 & 0\end{array}\right]$. Nevertheless, it needs to design a state observer for those whose state variables cannot be observed directly.

The membership functions are chosen from [27], and the initial reference is set as $(\mathrm{pi} / 7,0)$. Then combine the $M$ language with the truetime to carry out the simulation. The truetime is a toolbox of Matlab, which can offer a variety of communication network models. In addition, in the simulation, we set $\tau(k) T=2 T \sim 3 T$ and $d(k) T=T \sim 2 T$. The results of outputs of the wireless networked system in different situations are shown in Figure 3, where $y 1$ represents that there exists no time delay or packet dropout, $y 2$ shows that there are random time delay and packet dropout but no measures for compensation, and $y 3$ indicates the effect by using the compensation method proposed in this paper. It is easy to see that $y 3$ is stabilized faster than $y 2$. Consequently, 


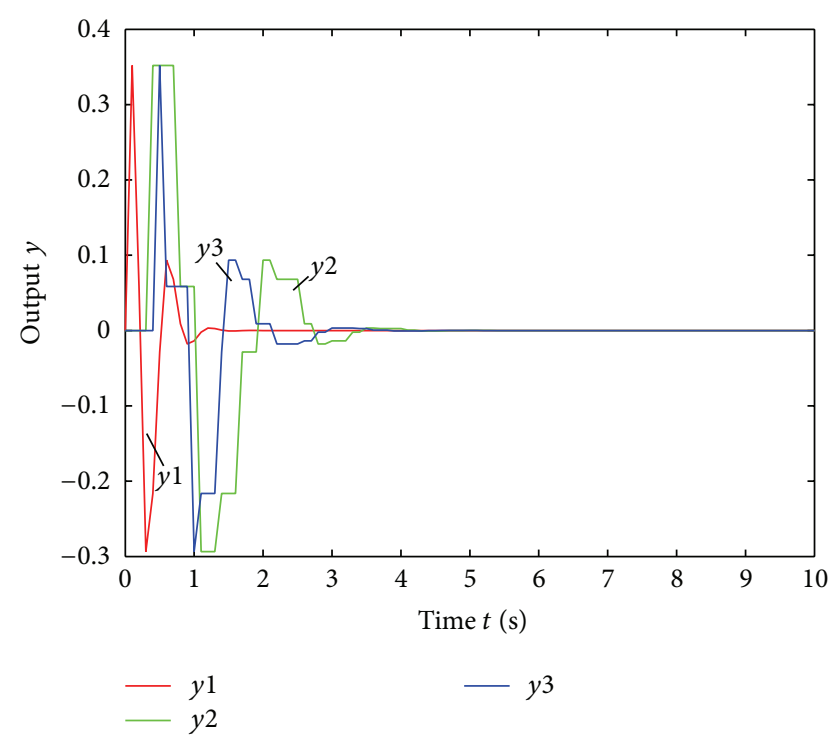

FIGURE 3: Outputs of the wireless networked system in different situations.

the approach has an advantage in T-S model-based wireless networked system with the random long time delay and data dropout issue.

\section{Conclusion}

Aimed at the wireless networked control systems with random time delay more than a sampling period and packet dropout, in this paper, on the basis of a compensation method in [19], we consider the inverted pendulum T-S fuzzy system as the plant and bring in counters that can deal with the packet dropout rate over the network. According to the separation principle, the state observation predictor and the state feedback controller are designed separately. Furthermore, there is a stability analysis for the closed-loop networked control systems with fixed time delay. A numerical example illustrates that the approach provided is effective in shortening long time delay and reducing the packet dropout in wireless networked system, which could be applied to the inverted pendulum T-S fuzzy system. The next step is to model the GPS and SI4432-based wireless sensor network (WSN) into T-S fuzzy system. Hence, the method mentioned in this paper can play a role in the WSN and then be applied in practice.

\section{Conflict of Interests}

The authors declare that there is no conflict of interests regarding the publication of this paper.

\section{Acknowledgments}

This work was supported by the National Natural Science Foundation of China under Grant no. 61374083, Science and Technology Department Project of Zhejiang Province under
Grant nos. 2014C31082, 2014C33109, and Program of Graduate Innovation Research in Zhejiang Sci-Tech University (YCX12028).

\section{References}

[1] R. Lu, Y. Xu, and A. Xue, " $H_{\infty}$ filtering for singular systems with communication delays," Signal Processing, vol. 90, no. 4, pp. 1240-1248, 2010.

[2] R. Q. Lu, H. Y. Wu, and J. J. Bai, "New delay-dependent robust stability criteria for uncertain neutral systems with mixed delays," Journal of the Franklin Institute: Engineering and Applied Mathematics, vol. 351, no. 3, pp. 1386-1399, 2014.

[3] R. Q. Lu, H. Li, and Y. P. Zhu, "Quantized $H_{\infty}$ filtering for singular time-varying delay systems with unreliable communication channel," Circuits, Systems, and Signal Processing, vol. 31, no. 2, pp. 521-538, 2012.

[4] H. Qian, B. Chen, and J. Yuan, "Enhanced routing protocol on AODV with load balance and delay restriction," Journal of Nanjing University of Science and Technology, vol. 37, no. 1, pp. 25-31, 2013.

[5] M. Xiang, Y. Xu, and Y. Zhang, "Dynamic routing strategy of wireless networks for construction crane inclination monitoring," Chinese Journal of Scientific Instrument, vol. 33, no. 9, pp. 1921-1930, 2012.

[6] Y. M. He and L. S. Wei, "Grey modeling and analysis for time-delay of star topology WNCSs," Computer Measurement \& Control, vol. 21, no. 3, pp. 732-734, 2013.

[7] J. Zhang, X. S. Xin, and H. B. Xu, "Output feedback control of a class of linear time-varying systems," Acta Automatica Sinica, vol. 40, no. 2, pp. 373-378, 2014.

[8] X. Zhao, S. Fei, and T. Li, "Quantized control for nonlinear singular impulsive systems with data dropouts," Control Theory and Applications, vol. 29, no. 4, pp. 539-543, 2012.

[9] R. Q. Lu, Y. Xu, A. K. Xue, and J. Zheng, "Networked control with state reset and quantized measurements: observer-based case," IEEE Transactions on Industrial Electronics, vol. 60, no. 11, pp. 5206-5213, 2013.

[10] R. Q. Lu, F. Wu, and A. K. Xue, "Networked control with reset quantized state based on bernoulli processing," IEEE Transactions on Industrial Electronics, vol. 61, no. 9, pp. 48384846, 2014.

[11] Y. Xu, H. Su, and Y. Pan, "Output feedback stabilization for Markov-based nonuniformly sampled-data networked control systems," Systems and Control Letters, vol. 62, no. 8, pp. 656-663, 2013.

[12] Z. Wu, P. Shi, H. Su, and J. Chu, "Passivity analysis for discretetime stochastic markovian jump neural networks with mixed time delays," IEEE Transactions on Neural Networks, vol. 22, no. 10, pp. 1566-1575, 2011.

[13] Z. G. Zheng, P. Shi, H. Y. Su, and J. Chu, "Stochastic synchronization of Markovian jump neural networks with time-varying delay using sampled-data," IEEE Transactions on Cybernetics, vol. 43, no. 6, pp. 1796-1806, 1806.

[14] Y. Yin, Y. Liu, and H. R. Karimi, "A simplified predictive control of constrained Markov jump system with mixed uncertainties," Abstract and Applied Analysis, vol. 2014, Article ID 475808, 7 pages, 2014.

[15] Y. Xu, H. Y. Su, Y. J. Pan, and Z. Wu, "Robust $H_{\infty}$ filtering for networked stochastic systems with randomly occurring sensor 
nonlinearities and packet dropouts," Signal Processing, vol. 93, no. 7, pp. 1794-1803, 2013.

[16] L. N. Rong, C. D. Yu, P. F. Guo, and H. Gao, "Fault detection for wireless networked control systems with stochastic uncertainties and multiple time delays," Abstract and Applied Analysis, vol. 2014, Article ID 605214, 6 pages, 2014.

[17] Z. Wu, P. Shi, H. Su, and J. Chu, "Asynchronous $l_{2}-l_{\infty}$ filtering for discrete-time stochastic Markov jump systems with randomly occurred sensor nonlinearities," Automatica, vol. 50, no. 1, pp. 180-186, 2014.

[18] Y. B. Luo, X. F. Jiang, and W. M. Zhong, "A fuzzy Lyapunov approach for constrained T-S fuzzy systems design," Control Theory \& Applications, vol. 27, no. 12, pp. 1777-1782, 2010.

[19] R. M. Wang, S. M. Fei, and L. Chai, "Predictive compensation for networked control systems," Control Theory \& Applications, vol. 28, no. 10, pp. 1473-1479, 2011.

[20] Y. F. Wang, Y. W. Jing, and S. Y. Zhang, "Fault-tolerant control for nonlinear networked control systems based on observer," Control Theory and Applications, vol. 29, no. 10, pp. 1348-1352, 2012.

[21] R. Lu, H. Wu, and J. Bai, "Networked $H_{\infty}$ filtering for T-S fuzzy systems with quantization and data dropouts," Journal of the Franklin Institute-Engineering and Applied Mathematics, vol. 351, no. 6, pp. 3126-3144, 2014.

[22] J. Kang and G. Z. Dai, "Design of networked control systems with state observer," Control and Decision, vol. 25, no. 6, pp. 943952, 2010.

[23] J. T. Feng, L. Wang, and F. Feng, "Analysis of linear timeinvariant discrete system operating under the state feedback control with observer," Journal of Electric Power, vol. 25, no. 5, pp. 411-418, 2010.

[24] X. N. Song and L. P. Liu, "Robust observer-based Ho control for nonlinear T-S fuzzy time-delay systems," Acta Physica Sinica, vol. 62, no. 21, pp. 490-500, 2013.

[25] K. Zheng, J. Xu, and L. Yu, "Takagi-Sugeno model-based optimal guaranteed cost fuzzy control for inverted pendulums," Control Theory and Applications, vol. 21, no. 5, pp. 703-708, 2004.

[26] H. B. Li, J. Q. Deng, Z. Q. Sun, and F. Sun, "Delay-dependent state feedback controller design for a class of networked control systems," Control Theory \& Applications, vol. 29, no. 10, pp. 13251330, 2012.

[27] B. Zhong and R. J. Zhan, "Sliding-mode control for second order nonlinear system based on generalized Gauss function," Computer Simulation, vol. 27, no. 4, pp. 371-374, 2010. 


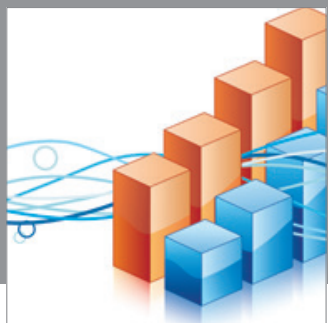

Advances in

Operations Research

mansans

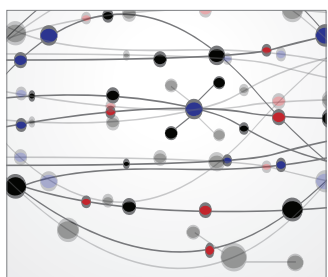

The Scientific World Journal
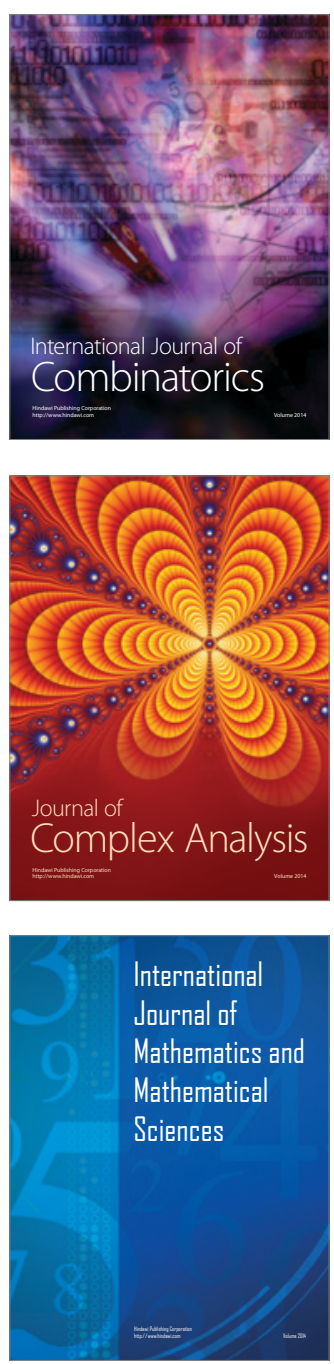
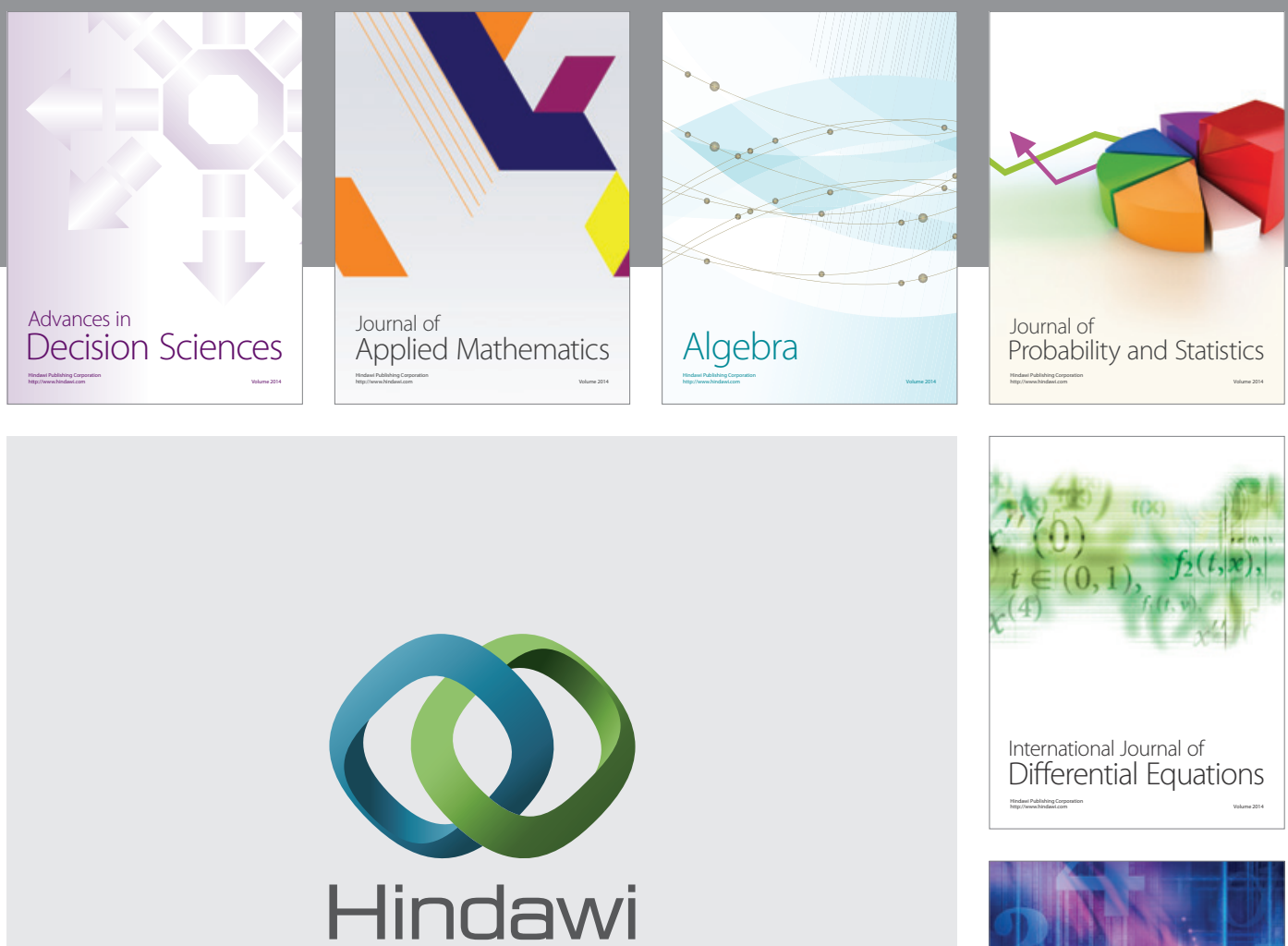

Submit your manuscripts at http://www.hindawi.com
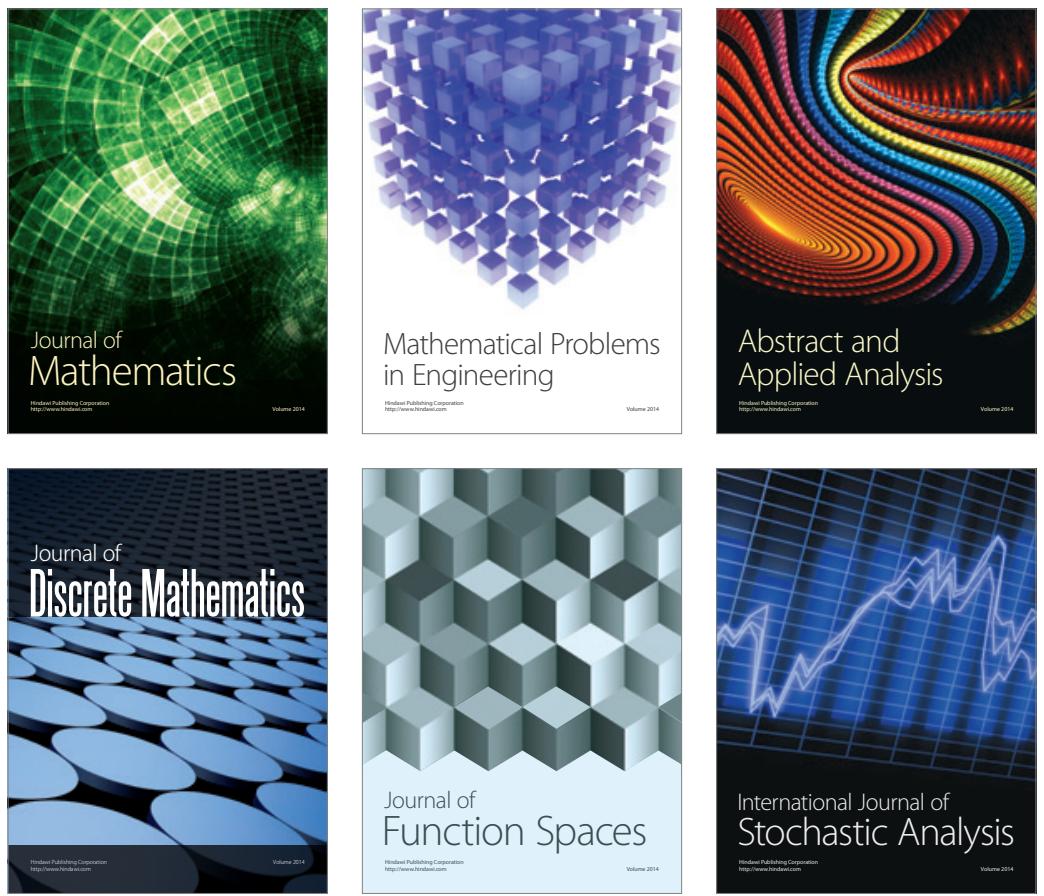

Journal of

Function Spaces

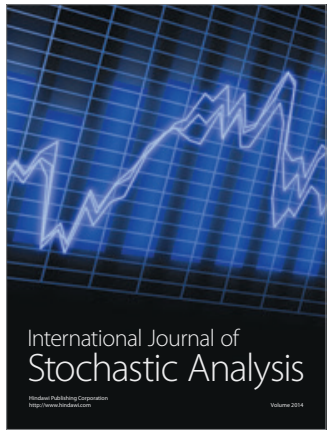

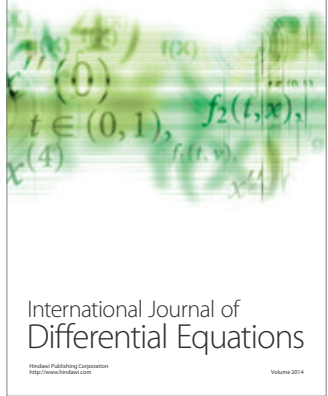
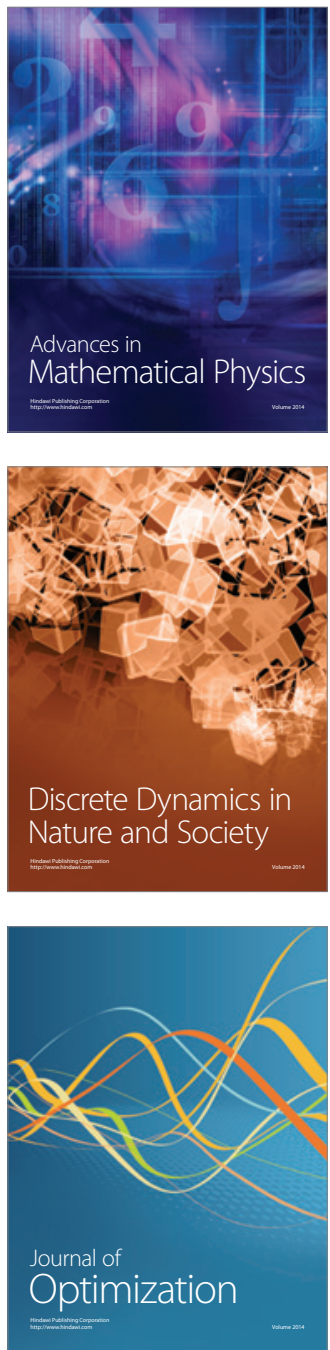